\title{
Confinement in the $q$-state Potts model: an RG-TCSA study
}

\author{
M. Lencsés ${ }^{a, b}$ and G. Takács ${ }^{a, c}$ \\ a MTA-BME "Momentum" Statistical Field Theory Research Group, \\ 1111 Budapest, Budafoki út 8, Hungary \\ ${ }^{b}$ Department of Theoretical Physics, Eötvös University, \\ 1117 Budapest, Pázmány Péter sétány 1/A, Hungary \\ ${ }^{c}$ Department of Theoretical Physics, Budapest University of Technology and Economics, \\ 1111 Budapest, Budafoki út 8, Hungary \\ E-mail: mate.lencses@gmail.com, takacsg@eik.bme.hu
}

ABSTRACT: In the ferromagnetic phase of the $q$-state Potts model, switching on an external magnetic field induces confinement of the domain wall excitations. For the Ising model $(q=2)$ the spectrum consists of kink-antikink states which are the analogues of mesonic states in QCD, while for $q=3$, depending on the sign of the field, the spectrum may also contain three-kink bound states which are the analogues of the baryons. In recent years the resulting "hadron" spectrum was described using several different approaches, such as quantum mechanics in the confining linear potential, WKB methods and also the BetheSalpeter equation. Here we compare the available predictions to numerical results from renormalization group improved truncated conformal space approach (RG-TCSA). While mesonic states in the Ising model have already been considered in a different truncated Hamiltonian approach, this is the first time that a precision numerical study is performed for the 3-state Potts model. We find that the semiclassical approach provides a very accurate description for the mesonic spectrum in all the parameter regime for weak magnetic field, while the low-energy expansion from the Bethe-Salpeter equation is only valid for very weak fields where it gives a slight improvement over the semiclassical results. In addition, we confirm the validity of the recent predictions for the baryon spectrum obtained from solving the quantum mechanical three-body problem.

KEYwords: Field Theories in Lower Dimensions, Exact S-Matrix, Integrable Field Theories

ARXIV EPRINT: 1506.06477 


\section{Contents}

1 Introduction 1

2 Confinement in $q$-state Potts field theory 2

2.1 Brief overview 2

2.2 Mesonic and baryonic mass estimations 4

2.2.1 Meson masses in the Ising model 4

2.2.2 Mesonic states in the 3-state Potts model 5

2.2.3 Baryon masses in the 3-state Potts model 7

3 TCSA, RG and extrapolation $\quad 7$

3.1 TCSA for the Ising and Potts field theories

3.1.1 Scaling Ising model $\quad 7$

3.1.2 Scaling 3-state Potts model 8

3.2 TCSA numerics: conventions 9

$\begin{array}{ll}3.3 & \text { Renormalization group improvement } \\ 3.4 & \text { Extrapolation }\end{array}$

$\begin{array}{lll}3.4 & \text { Extrapolation } & 11\end{array}$

4 Results 12

4.1 Testing ground: Ising model with magnetic field 12

$\begin{array}{lll}4.1 .1 & \text { False vacuum } & 12\end{array}$

$\begin{array}{lll}\text { 4.1.2 } & \text { Meson masses } & 16\end{array}$

4.2 3-state Potts model 16

$\begin{array}{lll}4.2 .1 & \text { False vacuum } & 18\end{array}$

$\begin{array}{lll}4.2 .2 & \text { Meson masses } & 18\end{array}$

$\begin{array}{lll}4.2 .3 & \text { Baryon masses } & 18\end{array}$

5 Conclusions $\quad 18$

\section{Introduction}

In this work we consider the scaling field theory corresponding to the $q$-state Potts model in the vicinity of the critical point separating the paramagnetic and ferromagnetic phases. In the absence of external magnetic field, the field theory in the ferromagnetic phase has a spectrum consisting of kinks (domain wall excitations) and is integrable with a known factorized $S$ matrix [1]. Switching on a weak external magnetic field induces a linear potential between the kinks, leading to their confinement.

In the case of the Ising model $(q=2)$ this scenario was proposed in [2], and has been investigated using several methods since then. One approach consists of a numerical Hamiltonian truncation method [3], which allows the numerical determination of the resulting 
meson spectrum. On the other hand, there are several approaches allowing predictions for the spectrum, with the first prediction already made in [2], which is in fact equivalent to finding the spectrum of quantum mechanical bound states in a linear potential. Form factor perturbation theory which treats the strength of integrability breaking (such as introduced by the magnetic field) as a small parameter can also be used to establish confinement $[4,5]$. The meson spectrum can also be computed using the WKB method [6, 7], and a low-energy expansion can be obtained from the Bethe-Salpeter equation [3, 6-9]. The validity of these approaches and their consistency has been established not only on the qualitative, but also at a quantitative level to high precision.

In the $q=3$ case the spectrum is expected to be richer and contain baryonic three-kink bound states besides the mesonic kink-antikink ones [10]. For the 3 -state Potts model, the Hamiltonian truncation method used in $[3,7]$ is not available, since it requires the knowledge of exact finite volume form factors of the magnetization operator in the off-critical theory in zero magnetic field. However, an alternative is provided by the truncated conformal space approach (TCSA) introduced in [11]. Since construction of low-energy spectrum in the TCSA does not depend on the assumption of integrability, nor on the existence of small parameters, it can be readily applied to non-integrable models, as performed for the $q=2$ and 3 cases in [12] and [13], respectively; for more recent applications to other non-integrable quantum field theories cf. $[14,15]$. In the case of the 3 -state Potts model predictions for the meson masses were obtained in [16], and a previous study [13] has already confirmed qualitatively the expected mesonic and baryonic spectra.

More recently quantum mechanical predictions for the baryon mass spectrum have also been obtained [17]; the results obtained in [16, 17] make it possible to investigate the spectra in more detail to make a precise quantitative verification of the theoretical predictions. For this purpose a more advanced implementation of TCSA is needed, which is based on the renormalization group improvements recently introduced and developed in [18-23]. In this work we discuss the application of this RG-TCSA method to the Ising and 3-state Potts models and compare it to the theoretical predictions.

The outline of the present paper is as follows. In section 2 we review the known results about confinement in the scaling $q$-state Potts field theory. In section 3 we briefly describe our TCSA implementation. Section 4 contains our results, starting with the Ising case as a testing ground for our numerical procedures and then turning to the 3-state Potts model. In section 5 we draw our conclusions.

\section{Confinement in $q$-state Potts field theory}

\subsection{Brief overview}

In this section we briefly overview the phenomenology of the $q$-state model based on [10]. The $q$-state Potts field theory is the scaling field theory of the $q$-state Potts model which is the generalization of the Ising model with $q$ different values (colours) of the lattice 
variables [24]. The lattice Hamiltonian can be written as

$$
\mathcal{H}=-\frac{1}{T} \sum_{\langle x, y\rangle} \delta_{s(x), s(y)}-H \sum_{x} \delta_{s(x), q}
$$

The first term is the standard nearest-neighbour interaction and second term is a generalized magnetic field in the "direction" of the $q$-th colour. Without magnetic field $(H=0)$ the theory has $\mathbb{S}_{q}$ permutation symmetry and has a critical temperature $T=T_{c}$, below which the system is in the ordered (ferromagnetic) phase while above $T_{c}$ the system is in the disordered (paramagnetic) phase.

In this work we consider the ferromagnetic phase, in which there are $q$ degenerate ground states (with all sites having the same colour), and the elementary excitations are kinks corresponding to domain walls. The action of the scaling field theory of the system can be written as a perturbation of the conformal field theory (CFT) corresponding to the critical point:

$$
S=S_{\mathrm{CFT}}^{(q)}+\tau \int d^{2} x \varepsilon(x)+h \int d^{2} x \sigma(x)
$$

where the couplings $\tau$ and $h$ are related to the lattice couplings

$$
\begin{aligned}
& \tau \sim T-T_{c} \\
& h \sim H
\end{aligned}
$$

and $x=\left(x_{1}, x_{2}\right)$ are Euclidean time and space coordinates. The corresponding CFT [25] is defined for $q \leq 4$ and has the central charge [26]

$$
c(q)=1-\frac{6}{t(t+1)}
$$

where the parameter $t$ is related to $q$ via the relation

$$
\sqrt{q}=2 \sin \frac{\pi(t-1)}{2(t+1)}
$$

and the thermal and magnetic fields $\varepsilon$ and $\sigma$ are identified with spinless relevant primaries $\Phi_{2,1}$ and $\Phi_{(t-1) / 2,(t+1) / 2}$ of the CFT and have scaling dimensions $[26,27]$

$$
\begin{aligned}
& 2 h_{\varepsilon}^{(q)}=X_{\varepsilon}^{(q)}=\frac{1}{2}\left(1+\frac{3}{t}\right) \\
& 2 h_{\sigma}^{(q)}=X_{\sigma}^{(q)}=\frac{(t-1)(t+3)}{8 t(t+1)}
\end{aligned}
$$

The thermal operator preserves the $\mathbb{S}_{q}$ symmetry while adding the magnetic field breaks the permutation symmetry according to $\mathbb{S}_{q} \rightarrow \mathbb{S}_{q-1}$.

In absence of the magnetic field the theory is integrable and the complete scattering theory is known $[1,28,29]$. In the ferromagnetic phase there exist $q$ degenerate ground states and the elementary excitations are kinks interpolating between them, with their mass $m$ related to the coupling $\tau$ via the mass gap relation [30]

$$
\tau=\kappa^{(q)} m^{2-2 h_{\varepsilon}^{(q)}}
$$


where

$$
\begin{aligned}
\kappa^{(2)} & =\frac{1}{2 \pi} \\
\kappa^{(3)} & =\frac{\Gamma\left(\frac{3}{10}\right)\left[\Gamma\left(\frac{2}{3}\right) \Gamma\left(\frac{5}{6}\right)\right]^{6 / 5}}{4 \times 2^{1 / 5} \pi^{8 / 5} \Gamma\left(\frac{7}{10}\right)} \sqrt{\frac{\Gamma\left(-\frac{1}{5}\right) \Gamma\left(\frac{7}{5}\right)}{\Gamma\left(-\frac{2}{5}\right) \Gamma\left(\frac{6}{5}\right)}}=0.1643033 \ldots
\end{aligned}
$$

In a magnetic field $h$ in the direction of the $q$-th colour the degeneracy between the $q$ ground states is lifted. For $h<0$ there is a single true vacuum and $q-1$ metastable ones, while for $h>0$ their roles are exchanged. The expectation values of the magnetic field operator in the direction of the $\gamma$-th colour is in the $\alpha$-th ground state of the zero field theory is written as

$$
\left\langle\sigma_{\gamma}\right\rangle_{\alpha} \equiv\left\langle 0_{\alpha}\left|\sigma_{\gamma}(x)\right| 0_{\alpha}\right\rangle=\frac{v^{(q)}}{q-1}\left(q \delta_{\gamma, \alpha}-1\right)
$$

where $v^{(q)}$ can be calculated using the formulas in [31]:

$$
\begin{aligned}
& v^{(2)}=1.3578383417 \cdots \times m^{1 / 8} \\
& v^{(3)}=1.9382577836 \cdots \times m^{2 / 15}
\end{aligned}
$$

As a result, the difference of the energy density between the false and the true vacua is given by [10]

$$
\Delta \varepsilon=\delta \varepsilon_{\alpha}-\delta \varepsilon_{q} \simeq h\left(\left\langle\sigma_{q}\right\rangle_{\alpha}-\left\langle\sigma_{q}\right\rangle_{q}\right)=-\frac{v^{(q)} q}{q-1} h \quad \alpha \neq q
$$

The combination $v^{(q)} q /(q-1)$ is denoted by $\beta^{(q)}$ and called the string tension; $\beta^{(q)}|h|$ gives the slope of the linear potential induced by the magnetic field.

\subsection{Mesonic and baryonic mass estimations}

Here we present the various known estimations for the masses in the confinement spectrum for the case of the Ising and the 3-state Potts model.

\subsubsection{Meson masses in the Ising model}

The first mass estimation is the one obtained by McCoy and Wu [2]

$$
m_{n}^{A i}=m\left(2+\lambda^{2 / 3} z_{n}\right)
$$

where $-z_{n}$ is the $n$th zero of the Airy function and $\lambda$ is the dimensionless ratio

$$
\lambda=\frac{\beta^{(2)}|h|}{m^{2}}
$$

This solution can be derived from the quantum mechanical system of two kinks in a linear potential. The quantum mechanical system also allows solutions corresponding to the zeros for the derivative of the Airy function; however, the corresponding wave-functions are symmetric and so forbidden due to the fermionic nature of the kinks. 
The WKB mass spectrum can be obtained by solving the quantization condition

$$
\begin{aligned}
\frac{\sinh \left(2 \vartheta_{n}\right)-2 \vartheta_{n}}{\lambda} & =2 \pi(n-1 / 4) \\
m_{n}^{\mathrm{WKB}} & =2 m \cosh \left(\vartheta_{n}\right)
\end{aligned}
$$

It can be improved further by adding higher corrections in $\lambda$ :

$$
\begin{aligned}
\sinh \left(2 \vartheta_{n}\right)-2 \vartheta_{n} & =2 \pi(n-1 / 4) \lambda+\sum_{k=1}^{\infty} \lambda^{k+1} S_{k}\left(\vartheta_{n}\right) \\
m_{n}^{\mathrm{iWKB}} & =2 m \cosh \left(\vartheta_{n}\right)
\end{aligned}
$$

The first term in this expansion is given in [7] and it is

$$
S_{1}(\vartheta)=\frac{1}{\sinh (2 \vartheta)}\left(-\frac{1}{6} \sinh ^{2}(\vartheta)+\frac{5}{24 \sinh ^{2}(\vartheta)}+\frac{1}{4 \cosh ^{2}(\vartheta)}-\frac{1}{12}\right)
$$

The Bethe-Salpeter equation (with various improvements) leads to a low energy expansion of the form

$$
\frac{\left(m_{n}^{l e}\right)^{2}}{4 m^{2}}=1+\sum_{k=1}^{\infty} \mu_{k} t^{k}
$$

with the parameter $t=\lambda^{2 / 3}$. Different approximations of the $\mu_{k}$ coefficients taking into account multi-quark corrections (such as quark mass renormalization and renormalization of the short range quark-antiquark interaction), and string tension renormalization can be found in [3, 6-8]. The low-energy expansion for the meson mass $\tilde{m}_{n}$ from the Bethe-Salpeter equation has the form [7]

$$
\begin{aligned}
\frac{\tilde{m}_{n}^{2}}{4 m^{2}}= & 1+z_{n} t^{2}+\frac{z_{n}^{2}}{5} t^{4}-\left(\frac{3 z_{n}^{3}}{175}+\frac{57}{280}\right) t^{6}+\left(\frac{23 z_{n}^{4}}{7875}+\frac{1543 z_{n}}{12600}\right) t^{8} \\
& +\frac{13}{1120 \pi} t^{9}+\left(-\frac{1894 z_{n}^{5}}{3031875}-\frac{23983 z_{n}^{2}}{242550}\right) t^{10}+\frac{3313 z_{n}}{10080} t^{11}+\ldots
\end{aligned}
$$

while the radiative corrections modify this expression according to

$$
\frac{m_{n}^{l e}-\tilde{m}_{n}}{m}=a_{2} t^{2}+\frac{z_{n}}{6}\left(4 c_{2}-a_{2}\right) t^{8}-\frac{B_{2}}{4} t^{9}+O\left(t^{10}\right)
$$

where

$$
a_{2}=0.0710809 \ldots \quad c_{2}=-0.003889 \ldots
$$

are the leading order quark mass and string tension renormalization corrections computed in [7] and

$$
B_{2}=0.8
$$

is the leading order interaction renormalization correction obtained in $[8,9]$.

\subsubsection{Mesonic states in the 3-state Potts model}

For this latter model, the Bethe-Salpeter has not been carried out (albeit the setting was established in [16]), so we quote the linear potential quantum mechanics and the WKB results. 
$\boldsymbol{h}<\mathbf{0}$. For this sign of the magnetic field there is a single stable vacuum and two metastable ones. The two-kink configurations are

$$
K_{3 \alpha}\left(\vartheta_{1}\right) K_{\alpha 3}\left(\vartheta_{2}\right)
$$

where 3 is the stable vacuum, while $\alpha=1,2$ are metastable vacua. Due to the presence of this degree of freedom allowed by $\alpha$ both antisymmetric and symmetric solutions are allowed. From the simple quantum mechanical picture of two-kink configurations it is clear that in the sector of zero total momentum the charge conjugation $(\mathcal{C})$ parity of kink-antikink and therefore also meson states is equal to their parity under spatial reflections.

The spectrum predicted by quantum mechanics in the linear potential is [16]

$$
\begin{aligned}
& m_{-, n}^{(A i)}=m\left(2+\lambda^{2 / 3} z_{n}\right)+\mathcal{O}\left(\lambda^{4 / 3}\right) \\
& m_{+, n}^{(A i)}=m\left(2+\lambda^{2 / 3} z_{n}^{\prime}\right)+\mathcal{O}\left(\lambda^{4 / 3}\right)
\end{aligned}
$$

where $\lambda$ is the dimensionless ratio

$$
\lambda=\frac{\beta^{(3)}|h|}{m^{2}}
$$

and $-z_{n}$ is the $n$-th zero of the Airy function, while $-z_{n}^{\prime}$ is the $n$-th zero of its derivative.

For this case, the WKB quantization is given by [16]

$$
\begin{aligned}
\frac{\sinh \left(2 \vartheta_{n}\right)-2 \vartheta_{n}}{\lambda} & =2 \pi\left(n-\frac{1}{4}\right)+2 \arctan \left(\frac{\tanh 2 \vartheta_{n}}{\sqrt{3}}\right)+i \mathcal{A}\left(2 \vartheta_{n}\right)+O(\lambda) \quad \text { odd } \\
\frac{\sinh \left(2 \vartheta_{n}\right)-2 \vartheta_{n}}{\lambda} & =2 \pi\left(n-\frac{3}{4}\right)+2 \arctan \left(\frac{\tanh 2 \vartheta_{n}}{\sqrt{3}}\right)+i \mathcal{A}\left(2 \vartheta_{n}\right)+O(\lambda) \quad \text { even } \\
\mathcal{A}(\vartheta) & =\log \left(\frac{\sinh (i \pi / 3+\vartheta)}{\sinh (i \pi / 3-\vartheta)}\right) \\
m_{ \pm, n}^{\mathrm{WKB}} & =2 m \cosh \left(\vartheta_{n}\right)
\end{aligned}
$$

This includes effects of nontrivial kink-antikink scattering, and therefore despite being semiclassical it goes beyond the simple quantum mechanical result above.

$\boldsymbol{h}>\mathbf{0}$. For this sign of the magnetic field there are two stable vacua and one metastable. The allowed two-kink configurations are

$$
K_{\alpha 3}\left(\vartheta_{1}\right) K_{3 \beta}\left(\vartheta_{2}\right)
$$

Charged meson states correspond to $\alpha \neq \beta$, and their spectrum is given by the WKB quantization condition [16] is given by

$$
\frac{\sinh \left(2 \vartheta_{n}\right)-2 \vartheta_{n}}{\lambda}=2 \pi\left(n-\frac{1}{4}\right)+i \mathcal{A}\left(2 \vartheta_{n}\right)+O(\lambda)
$$

Note that charged meson single-particle states do not satisfy periodic boundary conditions on a circle due to $\alpha \neq \beta$, therefore they cannot be observed in TCSA.

On the other hand, neutral meson states do not exist, as they easily decompose under the process

$$
K_{\alpha 3}\left(\vartheta_{1}\right) K_{3 \alpha}\left(\vartheta_{2}\right) \rightarrow K_{\alpha \beta}\left(\vartheta_{2}\right) K_{\beta \alpha}\left(\vartheta_{1}\right)
$$


where for $\alpha=1,2$ one has $\beta=2,1$, respectively. This process is allowed by the ChimZamolodchikov kink scattering amplitudes [1]; also note that the kinks mediating between the stable vacua 1,2 are not confined.

\subsubsection{Baryon masses in the 3-state Potts model}

For the case $h<0$ all the kinks are confined. As a result, one may have three-kink bound states of the form

$$
\begin{aligned}
& K_{31}\left(\vartheta_{1}\right) K_{12}\left(\vartheta_{2}\right) K_{23}\left(\vartheta_{3}\right) \\
& K_{32}\left(\vartheta_{1}\right) K_{21}\left(\vartheta_{2}\right) K_{13}\left(\vartheta_{3}\right)
\end{aligned}
$$

corresponding to baryons and antibaryons. Both of these particles have the same spectrum due to charge-conjugation symmetry, and can be modeled in the form of a quantum mechanical three-body system. The low energy estimates for the baryon masses were recently obtained by Rutkevich [17] with the result

$$
M_{n}^{ \pm}=m\left(3+\left(\beta^{(3)}|h| / m^{2}\right)^{2 / 3} \epsilon_{n}^{ \pm}\right)+\mathcal{O}\left(|h|^{4 / 3}\right)
$$

where the \pm correspond to parity under space reflection, with the following numerical values of $\epsilon$ for the first three states:

$$
\begin{array}{lll}
\epsilon_{1}^{+}=4.602 & \epsilon_{2}^{+}=5.912 & \epsilon_{3}^{+}=7.098 \\
\epsilon_{1}^{-}=6.650 & \epsilon_{2}^{-}=7.734 & \epsilon_{3}^{-}=8.753
\end{array}
$$

There are no baryons for $h>0$ as the kinks between the two stable vacua are not confined.

\section{TCSA, RG and extrapolation}

\subsection{TCSA for the Ising and Potts field theories}

\subsubsection{Scaling Ising model}

The Hilbert space of any conformal field theory can be decomposed into products of irreducible representations of the left and right moving Virasoro algebras, which can be specified by giving their left and right conformal weights as

$$
\mathcal{S}_{h, \bar{h}}=\mathcal{V}_{h} \otimes \mathcal{V}_{\bar{h}}
$$

and every such sector corresponds to a primary field $\Phi_{h, \bar{h}}$. For the Ising model with central charge $c=1 / 2$ the full Hilbert space is

$$
\begin{gathered}
\mathcal{H}^{(2)}=\mathcal{S}_{0,0} \oplus \mathcal{S}_{\frac{1}{2}, \frac{1}{2}} \\
\oplus \mathcal{S}_{\frac{1}{16}, \frac{1}{16}}
\end{gathered}
$$

where the sectors on the first line are even, the ones on the second line are odd. The Hamiltonian is

$$
H=H_{\mathrm{CFT}}^{(2)}+\tau \int d x \varepsilon+h \int d x \sigma
$$


where

$$
\varepsilon=\Phi_{\frac{1}{2}, \frac{1}{2}} \quad \sigma=\Phi_{\frac{1}{16}, \frac{1}{16}}
$$

Note that for this model the values $h$ and $-h$ are physically equivalent since they are related by the $\mathbb{Z}_{2}$ symmetry of the conformal field theory.

For the Ising model we used the following level cut-offs with the dimensions of the truncated Hilbert space indicated below:

\begin{tabular}{|c|c|c|c|c|c|c|c|c|c|c|}
\hline$n$ & 6 & 7 & 8 & 9 & 10 & 11 & 12 & 13 & 14 & 15 \\
\hline $\operatorname{dim}$ & 77 & 127 & 213 & 338 & 551 & 840 & 1330 & 1994 & 3023 & 4476 \\
\hline
\end{tabular}

\subsubsection{Scaling 3-state Potts model}

The scaling limit of Potts model at the critical point is a minimal conformal field theory with central charge $[25,32]$

$$
c=\frac{4}{5}
$$

The Hilbert space of the Potts model is the $D_{4}$ modular invariant [33]

$$
\mathcal{H}=\mathcal{H}_{0} \oplus \mathcal{H}_{+} \oplus \mathcal{H}_{-} \oplus \mathcal{H}_{1}
$$

where

$$
\begin{aligned}
\mathcal{H}_{0} & =\mathcal{S}_{0,0} \oplus \mathcal{S}_{\frac{2}{5}, \frac{2}{5}} \oplus \mathcal{S}_{\frac{7}{5}, \frac{7}{5}} \oplus \mathcal{S}_{3,3} \\
\mathcal{H}_{ \pm} & =\mathcal{S}_{\frac{1}{15}, \frac{1}{15}}^{ \pm} \oplus \mathcal{S}_{\frac{2}{3}, \frac{2}{3}}^{ \pm} \\
\mathcal{H}_{1} & =\mathcal{S}_{\frac{2}{5}, \frac{7}{5}} \oplus \mathcal{S}_{\frac{7}{5}, \frac{2}{5}} \oplus \mathcal{S}_{0,3} \oplus \mathcal{S}_{3,0}
\end{aligned}
$$

The $D_{4}$ conformal field theory is invariant under the permutation group $\mathbb{S}_{3}$ generated by two elements $\mathcal{Z}$ and $\mathcal{C}$ with the relations

$$
\mathcal{Z}^{3}=1 \quad \mathcal{C}^{2}=1 \quad \mathcal{C} \mathcal{Z C}=\mathcal{Z}^{-1}
$$

which have the signatures

$$
\operatorname{sign} \mathcal{Z}=+1 \quad \operatorname{sign} \mathcal{C}=-1
$$

The sectors in $\mathcal{H}_{0}$ of (3.7) are invariant under the action of the permutation group $\mathbb{S}_{3}$, the ones in $\mathcal{H}_{ \pm}$form the two-dimensional irreducible representation, which is characterized by the following action of the generators:

$$
\begin{aligned}
\mathcal{C}| \pm\rangle & = \pm|\mp\rangle \\
\mathcal{Z}| \pm\rangle & =\cos \left(\frac{2 \pi}{3}\right)| \pm\rangle \pm \sin \left(\frac{2 \pi}{3}\right)|\mp\rangle
\end{aligned}
$$

while those in $\mathcal{H}_{1}$ transform according to the signature representation of $\mathbb{S}_{3}$.

The Hamiltonian is given by

$$
H=H_{\mathrm{CFT}}^{(3)}+\tau \int d x \varepsilon+h \int d x \sigma
$$


where

$$
\varepsilon=\Phi_{\frac{2}{5}, \frac{2}{5}} \quad \sigma=\Phi_{\frac{1}{15}, \frac{1}{15}}^{+}
$$

In our considerations an important role will be played by charge conjugation parity: the even sector under $\mathcal{C}$ consists of $\mathcal{H}_{0}$ and $\mathcal{H}_{+}$, while the odd sector consists of $\mathcal{H}_{1}$ and $\mathcal{H}_{-}$, with the dimensions as a function of the level cut-off $n$ given below:

\begin{tabular}{|c|c|c|c|c|c|c|}
\hline$n$ & 6 & 7 & 8 & 9 & 10 & 11 \\
\hline dim, even sector & 634 & 1210 & 2426 & 4437 & 8258 & 14545 \\
\hline dim, odd sector & 816 & 1572 & 3039 & 5592 & 10121 & $(17904)$ \\
\hline
\end{tabular}

In the case of the odd sector the left/right descendent levels must be different for some of the fields the right in order to get spinless fields. In our convention, the truncation level is chosen to agree with the smaller of the descendent levels. We remark that in the even sector, our conformal basis at level cut-off $n=8$ exactly corresponds to the one used in the paper [13]. However for the odd sector, our prescription is different from the one in [13], which corresponds to choosing the level cut-off to agree with the higher one of the descendent levels. In the extrapolations we used level cut-offs from 6 to 11 in the even sector and from 6 to 10 in the odd sector.

\subsection{TCSA numerics: conventions}

The conformal Hamiltonian on a finite circle of circumference $R$ can be written as

$$
H_{\mathrm{CFT}}^{(q)}=\frac{2 \pi}{R}\left(L_{0}+\bar{L}_{0}-\frac{c}{12}\right)
$$

In our TCSA calculations we consider the zero momentum sectors for which $L_{0}-\bar{L}_{0}=0$, and impose a level cutoff on the spectrum of states according to

$$
\min \left(L_{0}-\Delta, \bar{L}_{0}-\bar{\Delta}\right) \leq n
$$

with $n$ a positive integer, where $\Delta, \bar{\Delta}$ denote the left/right conformal weights of the corresponding primary state.

The computations are all performed in units of the kink mass $m$ of the $h=0$ model, which means the volume is measured in dimensionless units $r=m R$ and the dimensionless energy levels are $e=E / m$. The finite volume energy levels are given as functions $e_{i}(r)$, with $i$ indexing the different levels; by convention the vacuum is taken to correspond to $i=0$. Since we are interested in the mass spectrum, the relevant quantities are the relative energy levels

$$
\tilde{e}_{i}(r)=e_{i}(r)-e_{0}(r)
$$

One can also introduce the scaling function defined by

$$
d_{i}(r)=\frac{r}{2 \pi} e_{i}(r)
$$

In the conformal field theory, the scaling function is a constant and is given by the eigenvalue of the operator

$$
L_{0}+\bar{L}_{0}-\frac{c}{12}
$$


corresponding to the given level. In the off-critical model the leading term of $e_{i}(r)$ for large $r$ is given by the bulk energy and is therefore generally linear (the Ising case is an exception with a logarithmic contribution), and so the scaling functions $d_{i}(r)$ grow as $r^{2}$. On the other hand, the bulk contribution is universal for all levels, and in the absence of certain ultraviolet divergences (which is the case for the models considered here, as none of the perturbing operators has conformal weight $\Delta \geq 3 / 4[20])$, the relative energy levels $\tilde{e}_{i}(r)$ go to a constant for large $r$.

\subsection{Renormalization group improvement}

To leading order in the level cutoff $n$, the cutoff dependence of the TCSA can be canceled by allowing the couplings to run according to renormalization group equations derived from second order perturbation theory. For a Hamiltonian of the form

$$
H=\frac{2 \pi}{R}\left(L_{0}+\bar{L}_{0}-\frac{c}{12}\right)+\sum_{a} \lambda_{a} \int_{0}^{R} d x \Phi_{a}(x)
$$

the leading RG equations are [20, 22]

$$
\begin{aligned}
\tilde{\lambda}_{c}(n)-\tilde{\lambda}_{c}(n-1) & =\sum_{a, b} \tilde{\lambda}_{a}(n) \tilde{\lambda}_{b}(n) C_{a b}^{c} \frac{n^{2 h_{a b c}-3}}{2 \Gamma\left(h_{a b c}\right)^{2}}(1+O(1 / n)) \\
\tilde{\lambda}_{a} & =\frac{\lambda_{a} R^{2-2 h_{a}}}{(2 \pi)^{1-2 h_{a}}} \\
h_{a b c} & =h_{a}+h_{b}-h_{c}
\end{aligned}
$$

where $C_{a b}^{c}$ are the CFT operator product expansion coefficients:

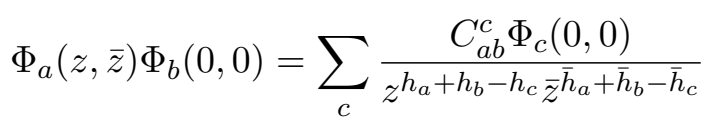

In the perturbation theory calculation, however, large denominators may appear due to the $r^{2}$ dependence of the scaling functions. This can be compensated for by taking into account the universal part of all scaling functions, by modifying the RG equations following the prescription in $[22,23]$

$$
\tilde{\lambda}_{c}(n)-\tilde{\lambda}_{c}(n-1)=\frac{1}{2 n-d_{0}(r)} \sum_{a, b} \tilde{\lambda}_{a}(n) \tilde{\lambda}_{b}(n) C_{a b}^{c} \frac{n^{2 h_{a b c}-2}}{\Gamma\left(h_{a b c}\right)^{2}}(1+O(1 / n))
$$

where the vacuum scaling function $d_{0}(r)$ can be estimated by its TCSA value at the starting cutoff for the RG run. In a unitary field theory it can be argued that the vacuum scaling function is always negative, so this does not introduce any new singularities. In essence this modification means regrouping some potentially large $1 / n$ corrections into the $\mathrm{RG}$ flow.

Note that this prescription also gives a running coupling for the identity, which leads to an additive renormalization constant for all energy levels. Since we consider only relative energy levels, this contribution can be omitted. 


\subsection{Extrapolation}

The higher $1 / n$ terms give state dependent corrections corresponding to non-local counter terms $[20,22,23]$. Their construction is quite involved and they are not known in a fully analytic form yet. However, there is an efficient shortcut that is sufficient for the purposes of the present work. The leading terms already incorporated in the RG equations yield cut-off corrections after integration with the scaling form

$$
n^{2 h_{a b c}-2}
$$

corresponding to the occurrence of $\Phi_{c}$ in the OPE $\Phi_{a} \Phi_{b}$.

In the Ising model, the leading exponents can be summarized as:

\begin{tabular}{|c|c|c|c|}
\hline$\Phi_{a} \Phi_{b} \backslash \Phi_{c}$ & $\mathbf{1}$ & $\sigma$ & $\epsilon$ \\
\hline$\sigma \sigma$ & $-\frac{7}{4}$ & - & $-\frac{11}{4}$ \\
\hline$\sigma \epsilon$ & - & -1 & - \\
\hline$\epsilon \epsilon$ & 0 & - & - \\
\hline
\end{tabular}

The exponent 0 corresponds to a logarithmic divergence in the ground state energy, which cancels from the relative energy levels. The exponent $-7 / 4$ also corresponds to ground state renormalization and therefore also cancels. The two other exponents are taken care of by the running couplings. Therefore it is only necessary to take into account the highest subleading $1 / n$ corrections, which lead to a residual cut-off dependence of the form

$$
\tilde{e}_{i}^{(n)}(r)=\tilde{e}_{i}(r)+\frac{A_{i}(r)}{n}+\frac{B_{i}(r)}{n^{2}}+O\left(n^{-11 / 4}\right)
$$

where both subleading terms come from $1 / n$ corrections to the $\epsilon \epsilon \mathbf{1}$ term.

In the Potts model, the leading exponents are

\begin{tabular}{|c|c|c|c|}
\hline$\Phi_{a} \Phi_{b} \backslash \Phi_{c}$ & $\mathbf{1}$ & $\sigma$ & $\epsilon$ \\
\hline$\sigma \sigma$ & $-\frac{26}{15}$ & $-\frac{28}{15}$ & $-\frac{38}{15}$ \\
\hline$\sigma \epsilon$ & - & $-\frac{6}{5}$ & - \\
\hline$\epsilon \epsilon$ & $-\frac{2}{5}$ & - & - \\
\hline
\end{tabular}

(where some fields contained in the OPE which give even higher exponents have been omitted) and the residual cut-off dependence is

$$
\tilde{e}_{i}^{(n)}(r)=\tilde{e}_{i}(r)+\frac{A_{i}(r)}{n^{7 / 5}}+\frac{B_{i}(r)}{n^{11 / 5}}+O\left(n^{-12 / 5}\right)
$$

Our prescription for the RG-TCSA is as follows. In units of $m, \tau$ is just a fixed dimensionless number given by $\kappa^{(q)}$. Therefore the TCSA has two dimensionless parameters, one of which is given by value of $h$ in units of $m$, i.e. the ratio

$$
\tilde{h}=h / m^{2-2 h_{\sigma}}
$$


and the dimensionless volume parameter $r=m R$. For any value of $r$ and $m$ the physical values of the perturbed CFT couplings are

$$
\begin{aligned}
& \tilde{\lambda}_{\epsilon}=-\frac{\kappa^{(q)} r^{2-2 h_{\epsilon}}}{(2 \pi)^{1-2 h_{\epsilon}}} \\
& \tilde{\lambda}_{\sigma}=\frac{h}{m^{2-2 h_{\sigma}}} \frac{r^{2-2 h_{\sigma}}}{(2 \pi)^{1-2 h_{\sigma}}}
\end{aligned}
$$

Taking these as initial conditions at $n=\infty$, the couplings can be run according to the RG equations (3.22) to determine their value at the given cut-off $n$. In practice one can approximate the difference equations by substituting

$$
\tilde{\lambda}_{c}(n)-\tilde{\lambda}_{c}(n-1) \rightarrow \frac{d \tilde{\lambda}_{c}}{d n}
$$

and solving the resulting differential equations numerically. We remark that in all our calculations the couplings ran very little so this has practically no effect, but as a matter of principle it must be done before we proceed to extrapolation. Once the RG eliminated all the leading cut-off dependencies, the renormalized TCSA Hamiltonian can be numerically diagonalized and then the residual cut-off dependence eliminated by fitting (3.24) for the Ising and (3.25) for the Potts case. In the case of the Ising model it turns out that the residual cut-off dependence alternates in sign between odd and even cut-offs, so the data for even and odd values of $n$ were fitted separately, as demonstrated in figure 1 . For the case of the Potts model no such alternation was observed, and the data could be reliably extrapolated including both even and odd values of the level cut-off $n$ as illustrated in 2 . We also remark that in the Potts case we took into account the two exponents indicated in (3.25); the $11 / 5$ and $12 / 5$ exponents are too close together, and their effect is to small compared to the leading $7 / 5$ to include both in the fit.

\section{Results}

\subsection{Testing ground: Ising model with magnetic field}

\subsubsection{False vacuum}

From the TCSA data it is possible to evaluate the energy density of the false vacuum relative to the stable one. For smaller volume, the TCSA converges fast, but for greater volumes the efficiency of the extrapolation procedure is apparent. The theoretical predictions can be calculated using (2.15) where the renormalized string tension given in [7] has also been taken into account. However, at the present precision the two predictions cannot be distinguished. Our results are illustrated in figure 3.

Note that the false vacuum level is not a continuous level in the volume, therefore its linear rise does not in fact contradict the statement that all relative energy levels $\tilde{e}_{i}(r)$ go to a constant for $r \rightarrow \infty$. The metastable states are seen as level avoidances in finite volume [12, 34]; this is best demonstrated by the false meson level in the Potts model, shown in subsection 4.2.2. For the false vacuum, however, the level avoidances are confined 


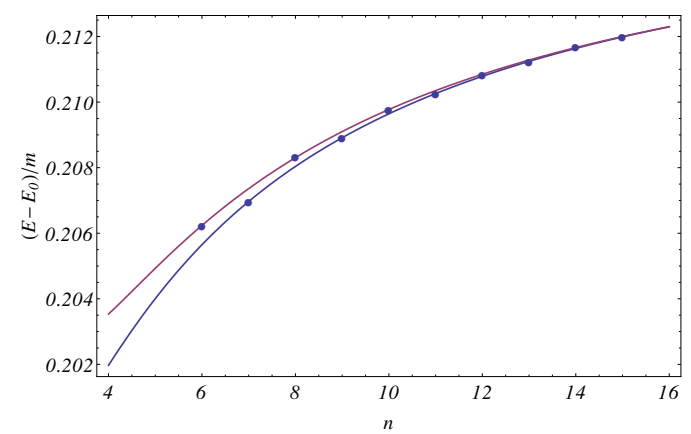

(a) First excited state

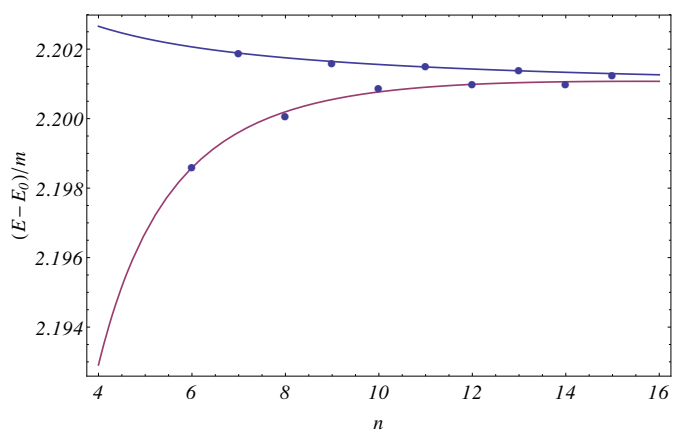

(b) Second excited state

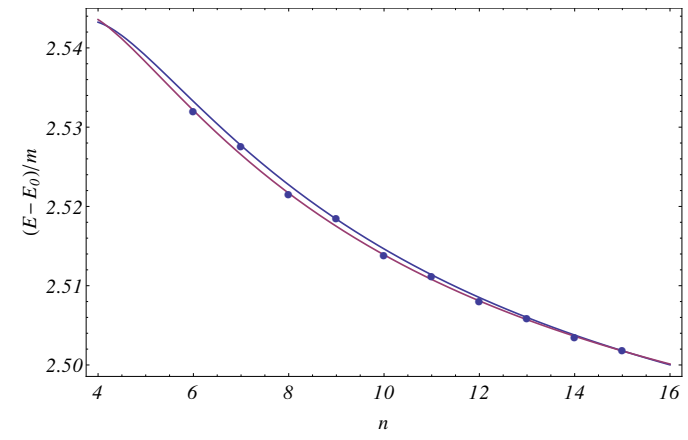

(c) Third excited state

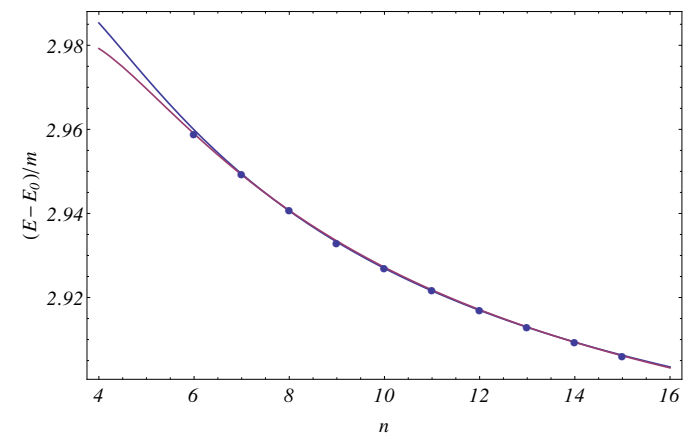

(d) Fourth excited state

Figure 1. Extrapolation fits of the relative energy levels in the Ising model in the ferromagnetic phase with magnetic field $\tilde{h}=0.008$ for the first four excited states at dimensionless volume $m R=10$. 


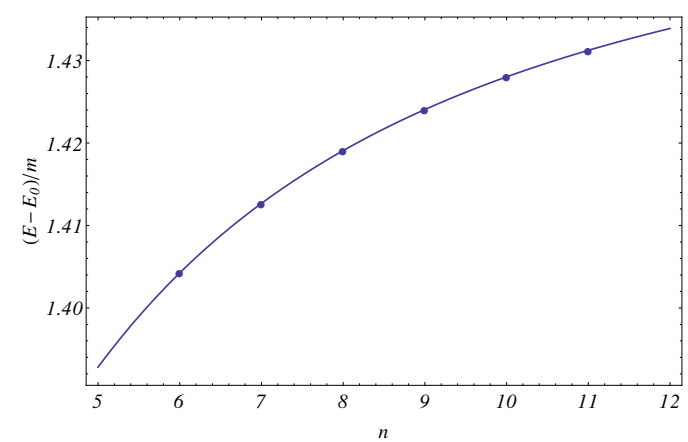

(a) First excited state

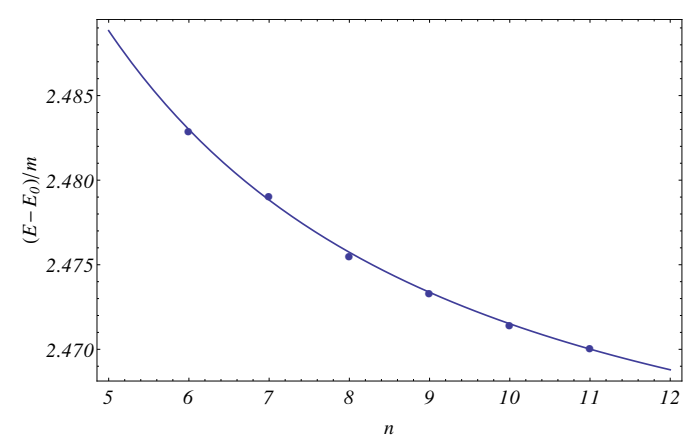

(b) Second excited state

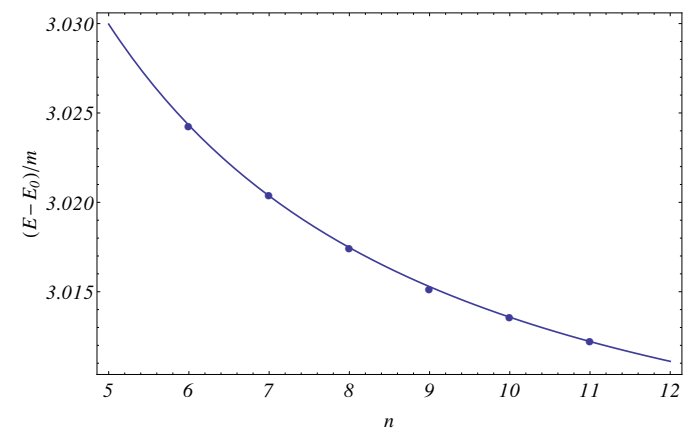

(c) Third excited state

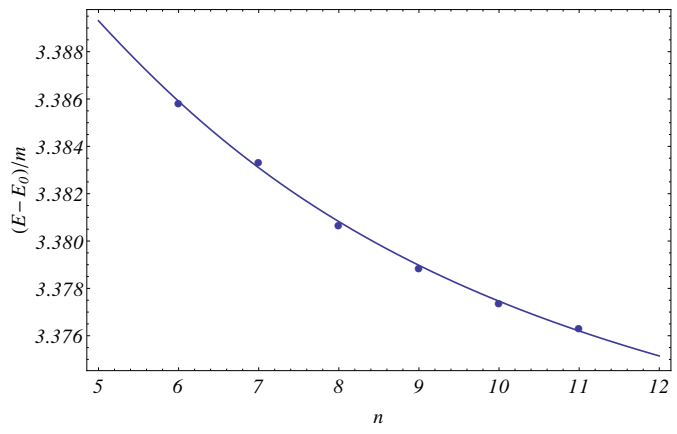

(d) Fourth excited state

Figure 2. Extrapolation fits of the relative energy levels in the Potts model in the ferromagnetic phase with magnetic field $\tilde{h}=-0.05$ for the first four excited states at dimensionless volume $m R=10$. 


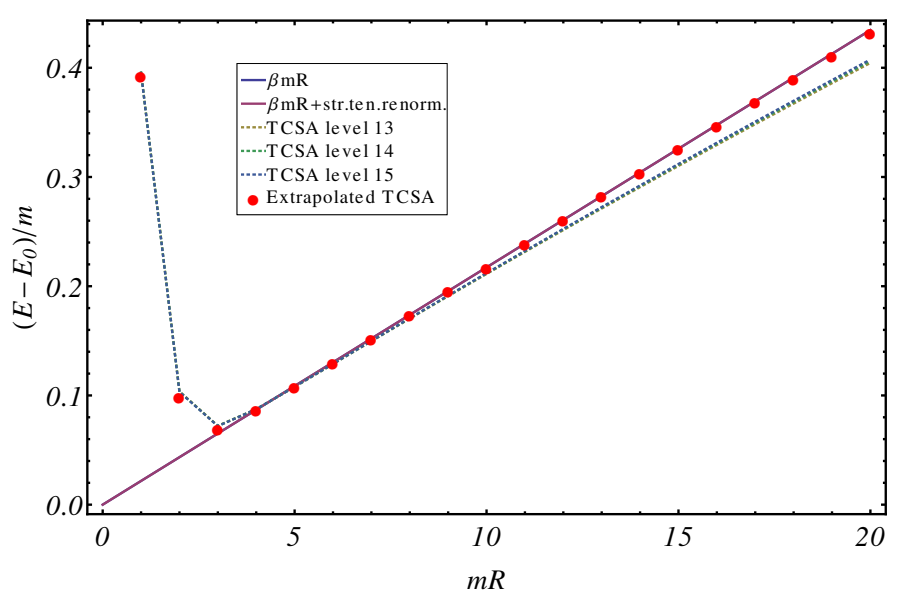

(a) $\tilde{h}=0.008$

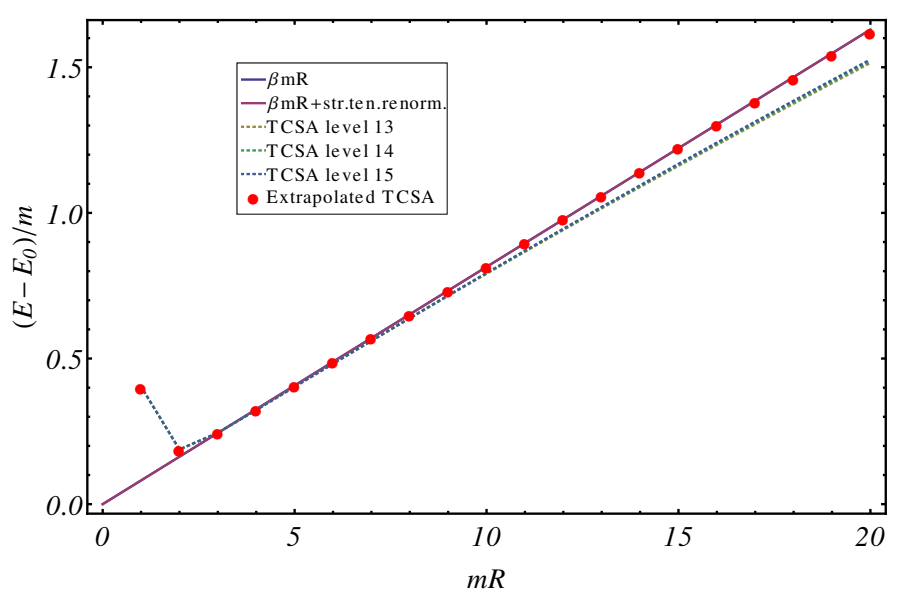

(b) $\tilde{h}=0.03$

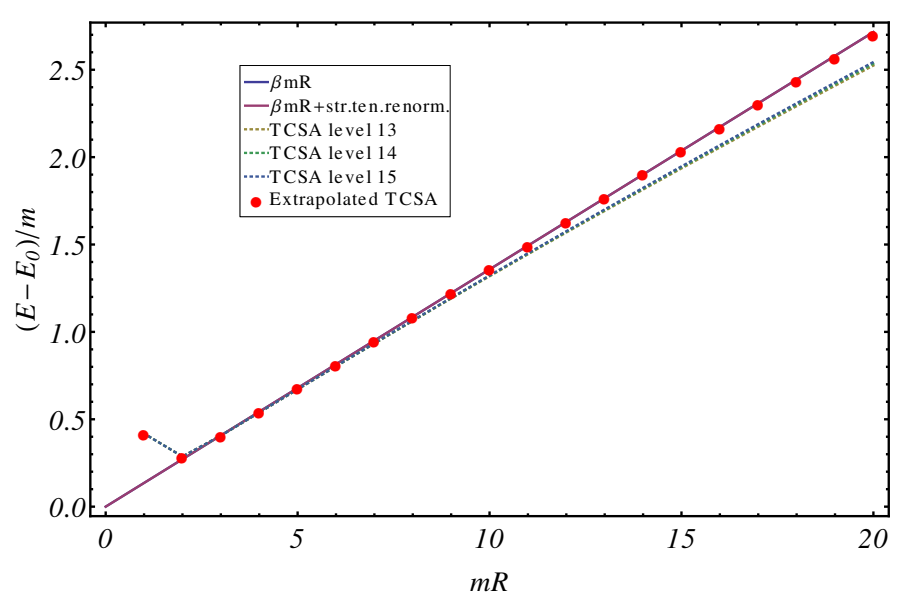

(c) $\tilde{h}=0.05$

Figure 3. Energy of the false vacuum for different values of the magnetic field in the Ising model. Lines are the theoretical predictions: blue is for (2.15), while purple one takes into account the string tension renormalization (the two lines are almost indistinguishable in the graphs). Dotted lines for different cut-off data, red dots are extrapolated (average of even and odd cut-off extrapolations). 
to small enough regions, so that the level can be glued together from the pieces. In addition, for small coupling the first level avoidance is at a much larger volume than shown in the plots.

\subsubsection{Meson masses}

The meson masses can be estimated by first extrapolating the levels separately for even and odd cut-offs, and taking the average of the two results. For a more precise measurement the numerical procedure was slightly modified by keeping only the $n=10 \ldots 15$ data and using only the $1 / n$ term in (3.24). The reason for this is that the meson level data do not allow fitting the $1 / n^{2}$ term with a sufficient precision.

Selecting a given extrapolated one-particle level, one then finds the volume where the level is the most flat. The data still contain exponential finite size effects, which can be suppressed by fitting the part of the extrapolated $n$th meson level just before the flat portion by an exponential function

$$
\tilde{e}_{n}(r)=m_{n}+A_{n} e^{-B_{n} r}
$$

and taking $m_{n}$ as the estimated mass of the $n$th meson. The results are shown in table 1 (we note that the data did not permit the volume extrapolation for $\tilde{h}=0.4$, so the TCSA numbers quoted there are just the value of the level at the point where it is most flat). The theoretical prediction "Airy" is given by eq. (2.16), while BS and WKB are given by (2.22) and (2.18), respectively; the last line (iWKB) corresponds to taking into account the first correction in (2.19). One can see that the prediction from simple quantum mechanics in a linear potential ("Airy") is generally only precise to a percent level or even worse for large magnetic fields, while WKB is an order of magnitude better. The theoretically rather involved Bethe-Salpeter approach only improves on WKB for very low mass mesons, while for higher masses the WKB is generally better. It is also clear that for $\tilde{h} \gtrsim 0.2$ the only working theoretical framework is the WKB method.

The upshot is that for all practical purposes WKB can be taken as the most reliable description of the spectrum over all the parameter range: it gives an approximation within $10^{-3}$ relative precision. This is an important lesson given that there are no Bethe-Salpeter predictions available in the 3-state Potts case yet; however, we can take the WKB as an accurate prediction for comparison with the 3 -state Potts meson data.

\subsection{3-state Potts model}

For the 3 -state Potts model we only consider the domain $h<0$, since according to the discussion in subsection 2.2.2 there are no meson one-particle levels for $h>0$, and reading off meson masses from two-particle states is difficult both for numerical reasons (the spectrum is dense, so level identification is difficult) and for theoretical reasons (extraction of masses with any precision requires modeling the meson-meson scattering). In addition, as discussed in subsection 2.2.3, there are no baryon states for $h>0$. 


\begin{tabular}{|c|c|c|c|c|}
\hline$\tilde{h}$ & & $m_{1}$ & $m_{2}$ & $m_{3}$ \\
\hline \multirow{5}{*}{0.0175} & TCSA & 2.303 & 2.526 & 2.71 \\
\hline & Airy & 2.3068 & 2.5364 & 2.7243 \\
\hline & BS & 2.3021 & 2.5228 & 2.7005 \\
\hline & WKB & 2.3000 & 2.5223 & 2.7003 \\
\hline & iWKB & 2.3011 & 2.5225 & 2.7004 \\
\hline \multirow{5}{*}{0.0250} & TCSA & 2.382 & 2.659 & 2.89 \\
\hline & Airy & 2.3891 & 2.6803 & 2.9187 \\
\hline & $\mathrm{BS}$ & 2.3816 & 2.6590 & 2.8813 \\
\hline & WKB & 2.3791 & 2.6583 & 2.8812 \\
\hline & iWKB & 2.3803 & 2.6586 & 2.8812 \\
\hline \multirow{5}{*}{0.0375} & TCSA & 2.497 & 2.855 & 3.15 \\
\hline & Airy & 2.5099 & 2.8915 & 3.2039 \\
\hline & BS & 2.4969 & 2.8556 & 3.1414 \\
\hline & WKB & 2.4941 & 2.8552 & 3.1418 \\
\hline & iWKB & 2.4954 & 2.8553 & 3.1417 \\
\hline \multirow{5}{*}{0.05} & TCSA & 2.597 & 3.027 & 3.37 \\
\hline & Airy & 2.6177 & 3.0800 & 3.4584 \\
\hline & BS & 2.5987 & 3.0281 & 3.3684 \\
\hline & WKB & 2.5958 & $\begin{array}{l}3.0283 \\
\end{array}$ & 3.3701 \\
\hline & iWKB & 2.5971 & 3.0282 & 3.3698 \\
\hline \multirow{5}{*}{0.1} & TCSA & 2.933 & 3.588 & 4.11 \\
\hline & Airy & 2.9805 & 3.7143 & 4.3151 \\
\hline & BS & 2.9312 & 3.5851 & 4.0919 \\
\hline & WKB & 2.9317 & 3.5946 & 4.1130 \\
\hline & iWKB & 2.9320 & 3.5933 & 4.1114 \\
\hline \multirow{5}{*}{0.2} & TCSA & 3.447 & 4.451 & 5.22 \\
\hline & Airy & 3.5565 & 4.7213 & 5.6750 \\
\hline & BS & 3.4115 & 4.3556 & 5.0251 \\
\hline & WKB & 3.4474 & 4.4508 & 5.2257 \\
\hline & iWKB & 3.4426 & 4.4455 & 5.2209 \\
\hline \multirow{5}{*}{0.4} & TCSA & 4.22 & 5.71 & 6.85 \\
\hline & Airy & 4.4707 & 6.3198 & 7.8337 \\
\hline & BS & 3.8964 & 4.8679 & 4.9503 \\
\hline & WKB & 4.2293 & 5.7277 & 6.8706 \\
\hline & iWKB & 4.2074 & 5.7102 & 6.8559 \\
\hline
\end{tabular}

Table 1. Meson masses in the Ising model. Theoretical predictions are shown with 4 digits accuracy, while for TCSA we show the digits that can be reliably extracted (with the last digit having an estimated precision of order 1). 


\subsubsection{False vacuum}

The relative energy of the false vacuum against the volume is shown in figure 4 . Notice that the extrapolation is again very effective.

\subsubsection{Meson masses}

In contrast to the Ising case here we can only use the method of extracting the value at the flattest portion of curve to estimate meson masses, due to the presence of the "false meson" resonance corresponding to a kink-antikink bound state configuration starting and ending in one of the false vacua. The "wavy" feature these resonance plateaus $[12,34]$ introduce in the spectrum prevent application of the exponential fit (4.1) to eliminate finite size effects in the meson mass. This effect can be seen in the plot 5 , which also demonstrates the efficiency of the numerical extrapolation procedure.

The mesonic spectrum against the absolute values of the magnetic field can be seen on figure 6. The deviations between the WKB prediction and the numerically determined masses are typically of the order of a few times $10^{-3}$, except in a few cases when a larger deviation of order $10^{-2}$ is observed. These are cases when the flattest portion of the meson level contains a level crossing with the false vacuum, which makes the truncation level extrapolation less precise.

\subsubsection{Baryon masses}

The baryonic states are in the higher part of the spectrum. As a result, because of the many level crossings the state must be carefully identified for each value of the volume and cut-off in order to carry out the extrapolation. The masses are extracted as the value of the extrapolated energy levels at its flattest point. As noted in subsection 2.2.3, baryons and antibaryons have the same spectra in infinite volume. In finite volume the eigenstates are the charge conjugation $(\mathcal{C})$ even and odd combinations, as can be seen from the results shown in figure 7 . The deviations between the theoretical prediction and the numerically determined masses are typically of the order of a few times $10^{-3}$, except in a few cases when a larger deviation of order a few times $10^{-2}$ is observed, which in this case is mostly due to difficulties of locating the level in the dense part of the spectrum.

\section{Conclusions}

In this work we investigated confinement in the $q$-state scaling Potts field theory, for the cases $q=2$ (Ising) and $q=3$ (3-state Potts). While these phenomena in the Ising model have been investigated in numerous works [3,6-9] since the seminal paper by McCoy and $\mathrm{Wu}[2]$, resulting in a very detailed understanding of the meson spectrum, in the case of the 3 -state Potts the theoretical predictions are more recent, especially regarding the baryon spectrum. Our method of choice was the renormalization group improved truncated conformal space approach (RG-TCSA), a Hamiltonian truncation method applied to perturbed conformal field theories, since there is no alternative for the 3 -state Potts model.

Ising model was used both as a benchmark of the method, and as a way of comparing the effectiveness of theoretical predictions. Our conclusion was that semiclassical (WKB) 


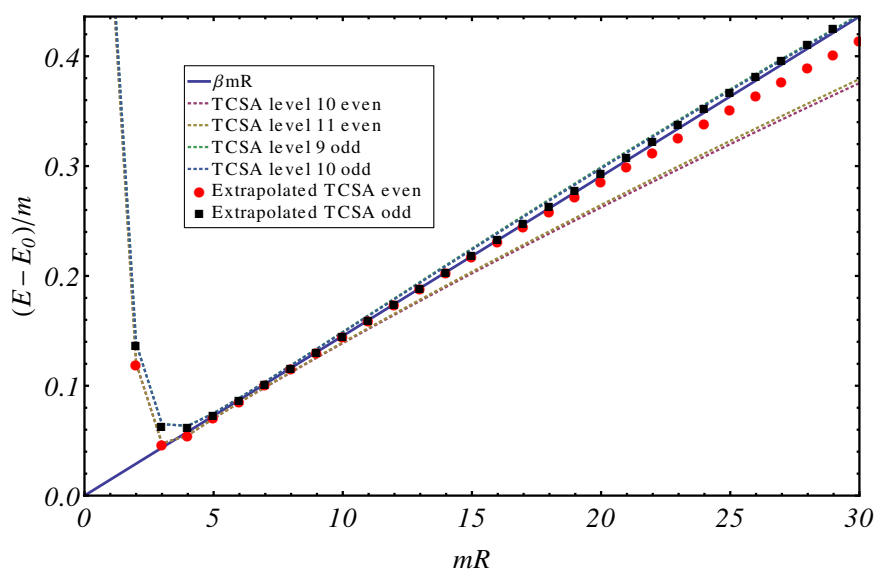

(a) $\tilde{h}=-0.005$

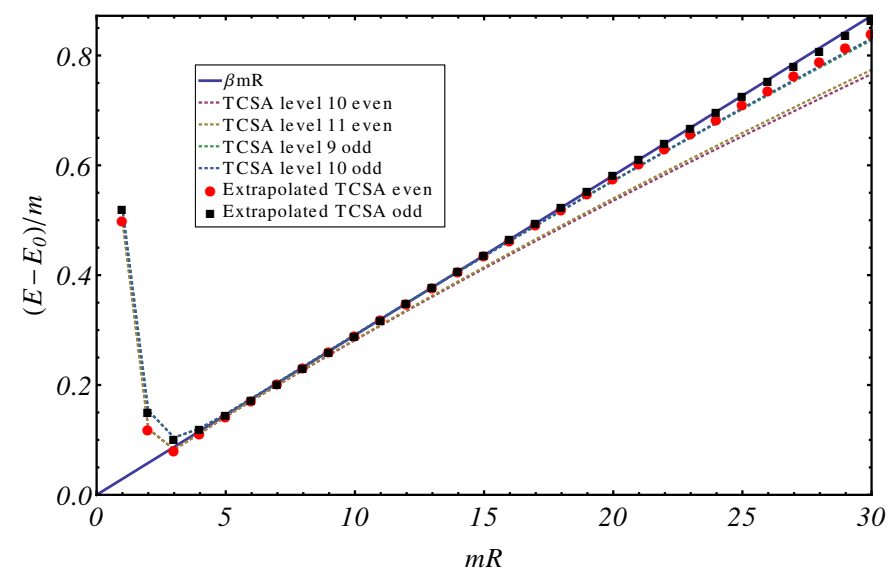

(b) $\tilde{h}=-0.01$

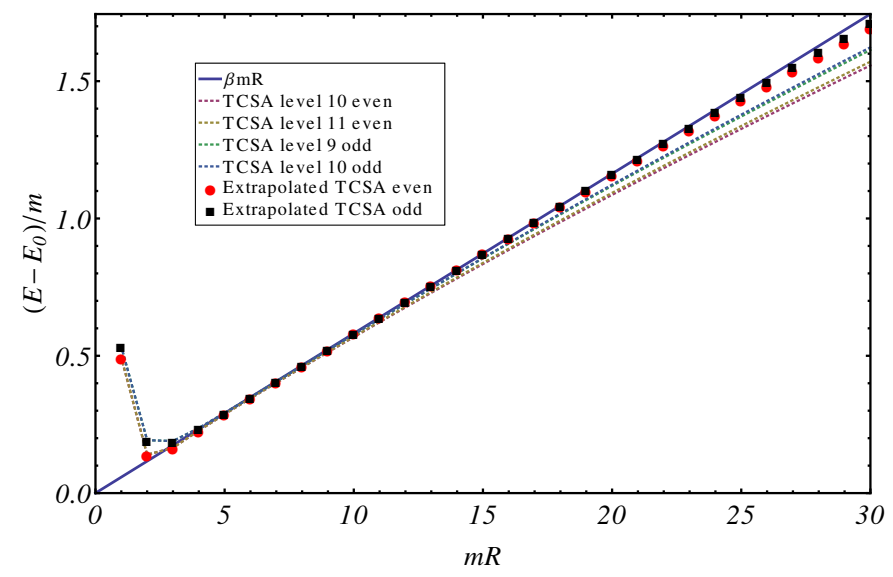

(c) $\tilde{h}=-0.02$

Figure 4. Energy of the false vacuum for different values of the magnetic field in the 3-state Potts model. Continuous lines are the theoretical predictions from (2.15), dashed lines are data with coupling constant renormalization before extrapolation for some values of the cut-off. Red dots are extrapolated data in the $\mathcal{C}$-even sector, while black squares are extrapolated data from the $\mathcal{C}$-odd sector. 


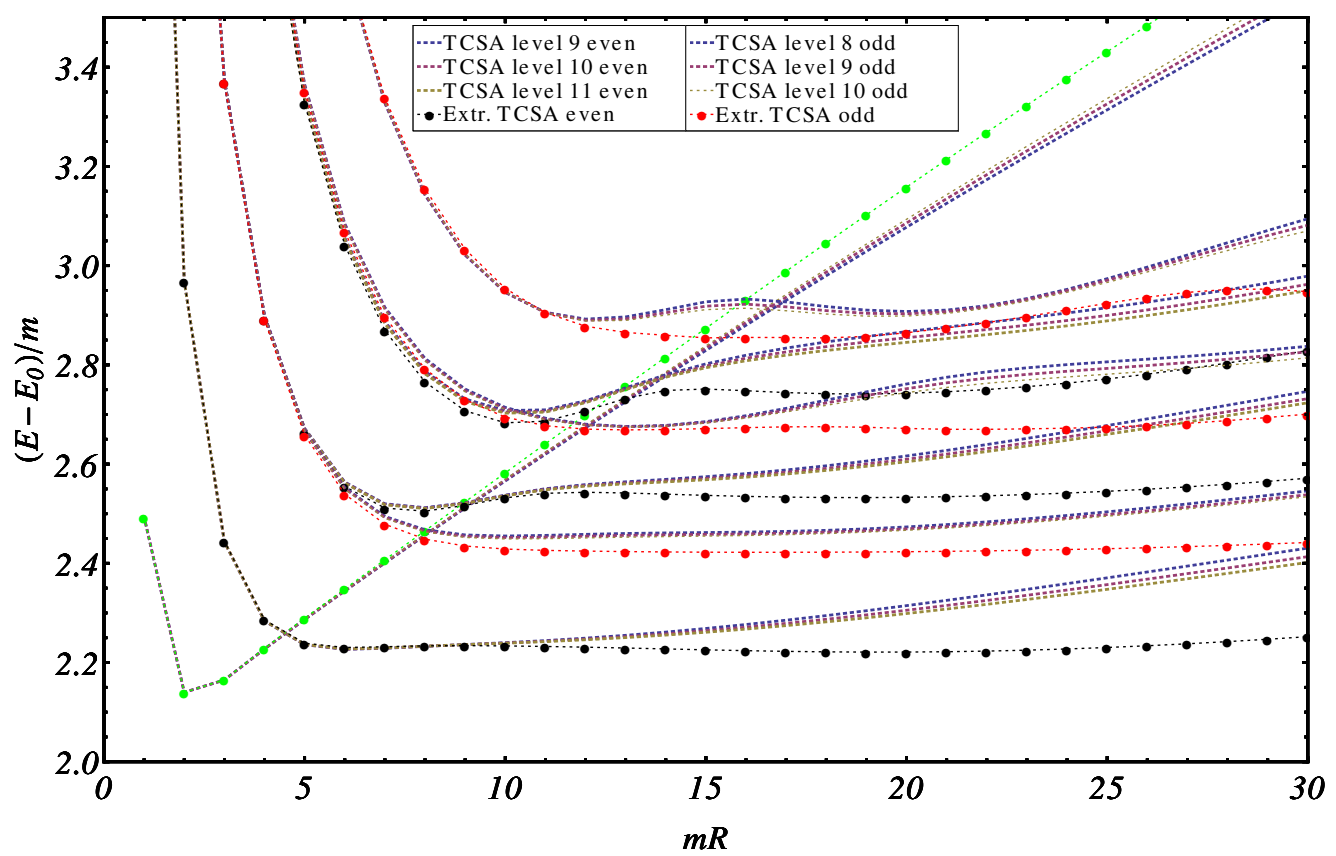

Figure 5. Effect of the extrapolation for the first three mesonic state in the $\mathcal{C}$-even and odd sectors with magnetic field $\tilde{h}=-0.02$. Dashed lines for data with running coupling for some values of the cut off, while large dots with dotted lines are the extrapolated data. We also plotted the energy of the even sector false vacuum (both before and after extrapolation, the latter marked with green) shifted up by $2 m$ to demonstrate that the wavy feature corresponds to the "false meson" resonance (meson configuration over the false vacuum).

quantization was efficient over all the range of weak magnetic field, and therefore could be taken as reference for the analysis of the Potts meson spectrum. Indeed, we could demonstrate very good agreement between the WKB and the meson spectra predicted in [16]. In addition, we compared the numerical results to recent predictions for the baryon spectrum [17], and found complete agreement.

The present results lead to the conclusions that from a quantitative phenomenological viewpoint, the meson spectrum of the the $q$-state scaling Potts field theory in weak magnetic field is described by WKB to a very high precision, while the baryon spectrum can be efficiently modeled by the three-body quantum mechanical model introduced in [17]. These findings are expected to be relevant in future investigations of the Potts field theory as a description of statistical systems.

\section{Acknowledgments}

The authors are grateful to S. Rutkevich for sharing his unpublished results in [9] and for discussions, and also to M. Mestyán for a careful reading of the manuscript. G.T. also acknowledges useful comments on the topic of RG-TCSA by S. Rychkov. This work was supported by the Momentum grant LP2012-50/2014 of the Hungarian Academy of Sciences. 


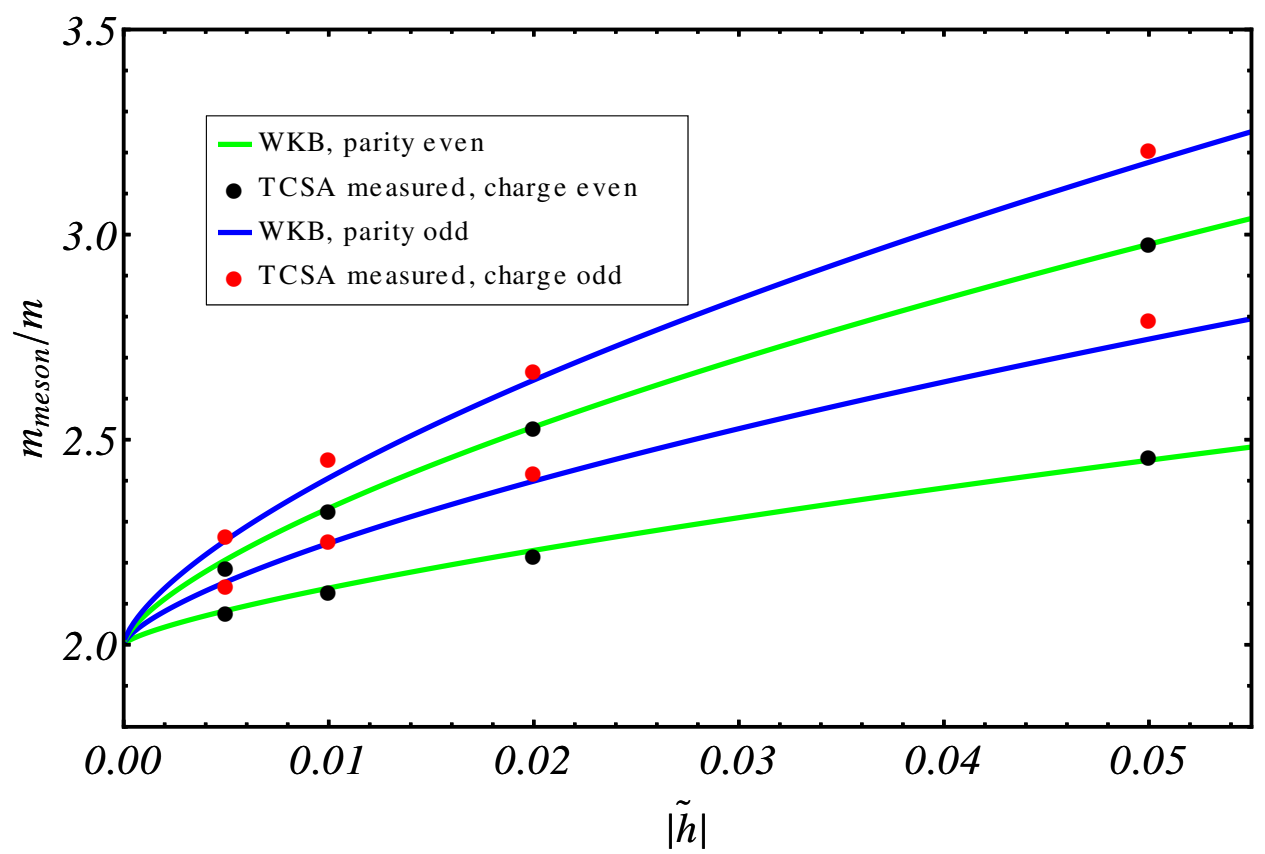

Figure 6. Meson masses against the magnetic field in the 3-state Potts model. Black dots show TCSA results from the $\mathcal{C}$-even sector while red is for $\mathcal{C}$-odd. The green and blue lines show the WKB predicted for parity even and odd states. As expected, for mesons the two parities coincide.

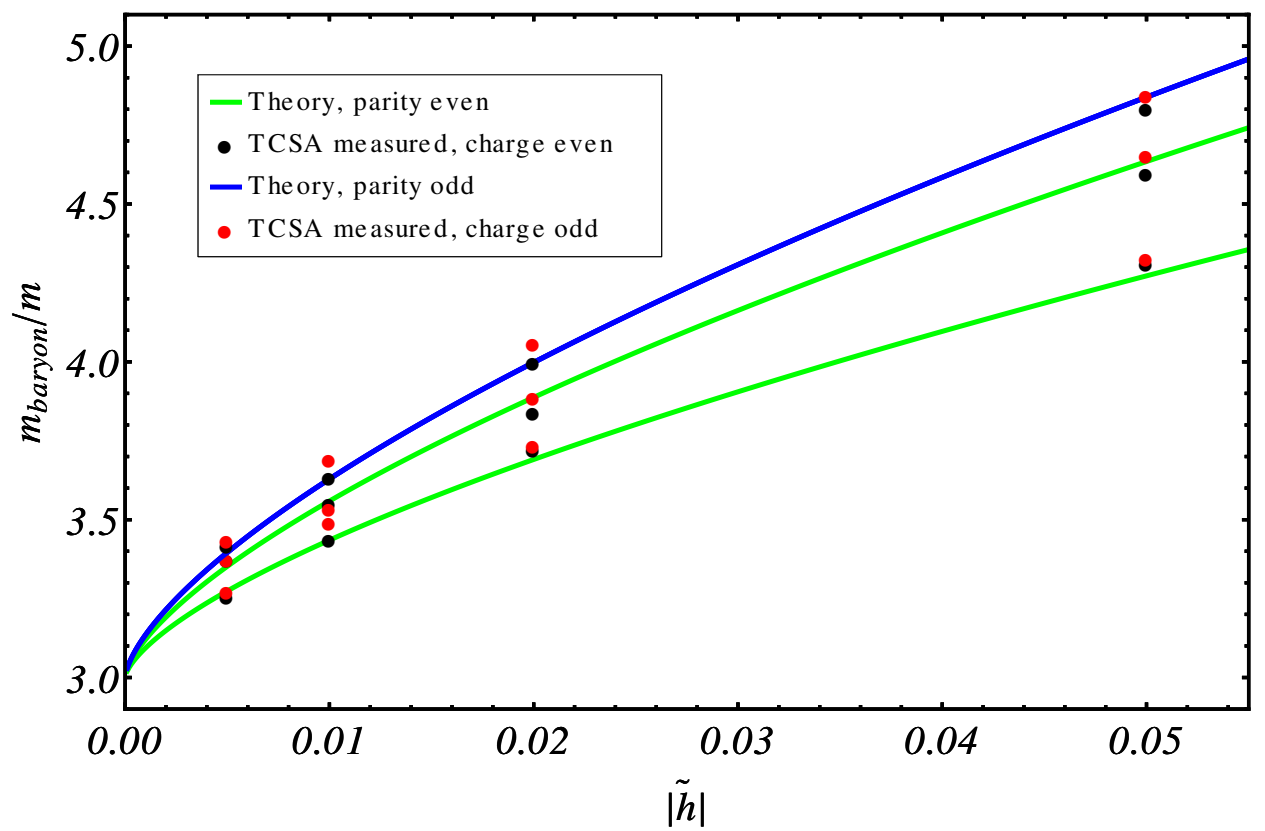

Figure 7. The three lowest baryon masses against the magnetic field in the 3-state Potts model. Black dots show TCSA results from the $\mathcal{C}$-even sector while red is for $\mathcal{C}$-odd. The green and blue lines show the predictions (2.33), (2.34) for space parity even and odd states, respectively. It can be seen that for each spatial parity there are two states, corresponding to the $\mathcal{C}$-even/odd combinations of the baryon with its antiparticle. 
Open Access. This article is distributed under the terms of the Creative Commons Attribution License (CC-BY 4.0), which permits any use, distribution and reproduction in any medium, provided the original author(s) and source are credited.

\section{References}

[1] L. Chim and A.B. Zamolodchikov, Integrable field theory of q-state Potts model with $0<q<4$, Int. J. Mod. Phys. A 7 (1992) 5317 [inSPIRE].

[2] B.M. McCoy and T.T. Wu, Two-dimensional Ising field theory in a magnetic field: breakup of the cut in the two point function, Phys. Rev. D 18 (1978) 1259 [InSPIRE].

[3] P. Fonseca and A.B. Zamolodchikov, Ising field theory in a magnetic field: analytic properties of the free energy, J. Stat. Phys. 110 (2003) 527.

[4] G. Delfino, G. Mussardo and P. Simonetti, Nonintegrable quantum field theories as perturbations of certain integrable models, Nucl. Phys. B 473 (1996) 469 [hep-th/9603011] [INSPIRE].

[5] G. Delfino and G. Mussardo, Nonintegrable aspects of the multifrequency sine-Gordon model, Nucl. Phys. B 516 (1998) 675 [hep-th/9709028] [INSPIRE].

[6] S.B. Rutkevich, Large- $N$ excitations in the ferromagnetic Ising field theory in a small magnetic field: mass spectrum and decay widths, Phys. Rev. Lett. 95 (2005) 250601 [hep-th/0509149] [INSPIRE].

[7] P. Fonseca and A. Zamolodchikov, Ising spectroscopy I: mesons at $T<T_{c}$, hep-th/0612304 [INSPIRE].

[8] S.B. Rutkevich, Formfactor perturbation expansions and confinement in the Ising field theory, J. Phys. A 42 (2009) 304025 [inSPIRE].

[9] S. Rutkevich, Confinement in Ising field theory and Ising spin chain: Bethe-Salpeter equation approach, talk presented at the Workshop on Quantum Matter in Low Dimensions: Opportunities and Challenge, Stockholm Sweden, 30 Aug-24 Sep 2010, http://agenda.albanova.se/getFile.py/access?contribId=265\&amp;resId=250\&amp; materialId $=$ slides\&amp; confId $=1128$.

[10] G. Delfino and P. Grinza, Confinement in the q-state Potts field theory, Nucl. Phys. B 791 (2008) 265 [arXiv:0706.1020] [INSPIRE].

[11] V.P. Yurov and A.B. Zamolodchikov, Truncated conformal space approach to scaling Lee-Yang model, Int. J. Mod. Phys. A 5 (1990) 3221 [InSPIRE].

[12] B. Pozsgay and G. Takács, Characterization of resonances using finite size effects, Nucl. Phys. B 748 (2006) 485 [hep-th/0604022] [INSPIRE].

[13] L. Lepori, G.Z. Toth and G. Delfino, The particle spectrum of the three-state Potts field theory: a numerical study, J. Stat. Mech. (2009) P11007 [arXiv:0909.2192] [INSPIRE].

[14] A. Coser, M. Beria, G.P. Brandino, R.M. Konik and G. Mussardo, Truncated conformal space approach for 2D Landau-Ginzburg theories, J. Stat. Mech. (2014) P12010 [arXiv: 1409.1494] [INSPIRE].

[15] R.M. Konik, T. Pálmai, G. Takács and A.M. Tsvelik, Studying the perturbed Wess-Zumino-Novikov-Witten $\mathrm{SU}(2)_{k}$ theory using the truncated conformal spectrum approach, Nucl. Phys. B 899 (2015) 547 [arXiv:1505.03860] [INSPIRE]. 
[16] S.B. Rutkevich, Two-kink bound states in the magnetically perturbed Potts field theory at $T<T_{c}$, J. Phys. A 43 (2010) 235004 [arXiv:0907.3697] [InSPIRE].

[17] S.B. Rutkevich, Baryon masses in the three-state Potts field theory in a weak magnetic field, J. Stat. Mech. (2015) P01010 [arXiv:1408.1818] [INSPIRE].

[18] G. Feverati, K. Graham, P.A. Pearce, G.Z. Tóth and G.M.T. Watts, A renormalization group for the truncated conformal space approach, J. Stat. Mech. (2008) P03011.

[19] R.M. Konik and Y. Adamov, Numerical renormalization group for continuum one-dimensional systems, Phys. Rev. Lett. 98 (2007) 147205.

[20] P. Giokas and G. Watts, The renormalisation group for the truncated conformal space approach on the cylinder, arXiv:1106.2448 [INSPIRE].

[21] M. Lencsés and G. Takács, Excited state TBA and renormalized TCSA in the scaling Potts model, JHEP 09 (2014) 052 [arXiv: 1405.3157] [INSPIRE].

[22] M. Hogervorst, S. Rychkov and B.C. van Rees, Truncated conformal space approach in d dimensions: a cheap alternative to lattice field theory?, Phys. Rev. D 91 (2015) 025005 [arXiv: 1409.1581] [INSPIRE].

[23] S. Rychkov and L.G. Vitale, Hamiltonian truncation study of the $\varphi^{4}$ theory in two dimensions, Phys. Rev. D 91 (2015) 085011 [arXiv:1412.3460] [INSPIRE].

[24] R.B. Potts, Some generalized order-disorder transformations, Math. Proc. Cambr. Phil. Soc. 48 (1952) 106 [inSPIRE].

[25] A.A. Belavin, A.M. Polyakov and A.B. Zamolodchikov, Infinite conformal symmetry in two-dimensional quantum field theory, Nucl. Phys. B 241 (1984) 333 [INSPIRE].

[26] V.S. Dotsenko and V.A. Fateev, Conformal algebra and multipoint correlation functions in two-dimensional statistical models, Nucl. Phys. B 240 (1984) 312 [INSPIRE].

[27] B. Nienhuis, Critical behavior of two-dimensional spin models and charge asymmetry in the Coulomb gas, J. Stat. Phys. 34 (1984) 731 [InSPIRE].

[28] R. Koberle and J.A. Swieca, Factorizable Z(N) models, Phys. Lett. B 86 (1979) 209 [INSPIRE].

[29] F.A. Smirnov, Exact S-matrices for $\phi_{1,2}$-perturbated minimal models of conformal field theory, Int. J. Mod. Phys. A 6 (1991) 1407 [inSPIRE].

[30] V.A. Fateev, The exact relations between the coupling constants and the masses of particles for the integrable perturbed conformal field theories, Phys. Lett. B 324 (1994) 45 [INSPIRE].

[31] V. Fateev, S.L. Lukyanov, A.B. Zamolodchikov and A.B. Zamolodchikov, Expectation values of local fields in Bullough-Dodd model and integrable perturbed conformal field theories, Nucl. Phys. B 516 (1998) 652 [hep-th/9709034] [INSPIRE].

[32] V.S. Dotsenko, Critical behavior and associated conformal algebra of the $Z_{3}$ Potts model, Nucl. Phys. B 235 (1984) 54 [inSPIRE].

[33] A. Cappelli, C. Itzykson and J.B. Zuber, Modular invariant partition functions in two-dimensions, Nucl. Phys. B 280 (1987) 445 [inSPIRE].

[34] M. Lüscher, Signatures of unstable particles in finite volume, Nucl. Phys. B 364 (1991) 237 [INSPIRE]. 